\title{
A massive graviton in topologically new massive gravity
}

\author{
Yong-Wan Kim, $1, *$ Yun Soo Myung, $2,+$ and Young-Jai Park ${ }^{3,+}$ \\ ${ }^{1}$ Center for Quantum Spacetime, Sogang University, Seoul 121-742, Korea \\ ${ }^{2}$ Institute of Basic Science and School of Computer Aided Science, \\ Inje University, Gimhae 621-749, Korea \\ ${ }^{3}$ Department of Physics and Department of Global Service Management, \\ Sogang University, Seoul 121-742, Korea
}

\begin{abstract}
We investigate the topologically new massive gravity in three dimensions. It turns out that a single massive mode is propagating in the flat spacetime, comparing to the conformal Chern-Simons gravity which has no physically propagating degrees of freedom. Also we discuss the realization of the BMS/GCA correspondence.

PACS numbers: 04.60.Rt, 04.20.Ha, 11.25.Tq

Keywords: Topologically New Massive Gravity; BMS/GCA correspondence
\end{abstract}

*Electronic address: ywkim65@gmail.com

${ }^{\dagger}$ Electronic address: ysmyung@inje.ac.kr

${ }^{\ddagger}$ Electronic address: yjpark@sogang.ac.kr 


\section{INTRODUCTION}

It is well known that the AdS/CFT correspondence [1] was supported by the observation that the asymptotic symmetry group of $\mathrm{AdS}_{3}$ spacetime is two-dimensional conformal symmetry group (two Virasoro algebras) on the boundary [2]. Similarly, the asymptotic symmetry group of flat spacetime is the infinite dimensional Bondi-Metzner-Sachs (BMS) group whose dual CFT is described by the Galilean conformal algebras (GCA). The latter was called the BMS/GCA correspondence [3]. The centrally extended BMS (or GCA) algebra is generated by two kinds of generators $L_{n}$ and $M_{n}$ :

$$
\begin{aligned}
& {\left[L_{m}, L_{n}\right]=(m-n) L_{n+m}+\frac{c_{1}}{12}\left(n^{3}-n\right) \delta_{n+m, 0},} \\
& {\left[L_{m}, M_{n}\right]=(m-n) M_{n+m}+\frac{c_{2}}{12}\left(n^{3}-n\right) \delta_{n+m, 0}} \\
& {\left[M_{m}, M_{n}\right]=0 .}
\end{aligned}
$$

It is very important to establish the BMS/GCA correspondence by choosing a concrete model. Recently, a holographic correspondence between a conformal Chern-Simon gravity (CSG) in flat spacetime and a chiral conformal field theory was reported in [4]. Choosing the CSG as the flat-spacetime limit of the topologically massive gravity (TMG) in the scaling limit of $\mu \rightarrow 0$ and $G \rightarrow \infty$ while $G \mu$ fixed, the BMS central charges are determined to be $c_{1}=24 k(=3 / G \mu)$ and $c_{2}=0$. This implies that the CSG is dual to a chiral half of a CFT with $c=24 k$. On the other hand, $c_{1}=0$ and $c_{2}=3 / G$ was predicted when using the Einstein gravity without taking the scaling limit [5].

Considering the flat spacetime expressed in terms of outgoing Eddington-Finkelstein (EF) coordinates, the linearized equation of the CSG leads to the third order equation $(D h)^{3}=0$. The solution to the first order equation $\left(D h^{\xi}\right)=0$ is given by [4]

$$
h_{\mu \nu}^{\xi}=e^{-i(\xi+2) \theta} r^{-(\xi+2)}\left(m_{1} \bigotimes m_{1}\right)
$$

where $\xi$ is the eigenvalue of $L_{0}$ and a Killing vector of $m_{1}=i e^{i \theta}\left(\partial_{u}-\partial_{r}-\frac{i}{r} \partial_{\theta}\right)$. Furthermore, one solution to $(D h)^{3}=0$ is given by $h_{\mu \nu}^{\log }=-i(u+r) h_{\mu \nu}^{\xi}$, while the other is $h_{\mu \nu}^{\log ^{2}}=$ $-\frac{1}{2}(u+r)^{2} h_{\mu \nu}^{\xi}$. These are the flat-space analogues of $\log$ - and $\log ^{2}$-solutions on the $\mathrm{AdS}_{3}$ spacetime.

At this stage, we wish to point out that the solutions $\left\{h^{\xi}, h^{\log }, h^{\log ^{2}}\right\}$ could not represent any physical modes propagating on the flat spacetime background because the CSG has no 
physical degrees of freedom. Actually, these all belong to the gauge degrees of freedom. Hence, it urges to find a relevant action which has a physically massive mode propagating on the Minkowski spacetime. This might be found when including a curvature square combination $K$, leading to the topologically new massive gravity (TNMG) [6]. The TNMG is also obtained from the generalized massive gravity (GMG) with two different massive modes [7, 8] when turning off the Einstein-Hilbert term and cosmological constant. If the Einstein-Hilbert term is omitted, it is called the cosmological TNMG [9]. It turned out that the linearized TNMG provides a single spin-2 mode with mass $\frac{m^{2}}{\mu}$ in the Minkowski spacetime, which becomes a massless mode of massless NMG in the limit of $\mu \rightarrow \infty[6,10]$. Very recently, it was argued that this reduction $(2 \rightarrow 1)$ of local degrees of freedom is an artefact of the linearized approximation by using the Hamiltonian formulation where nonlinear effect is not ignored [11]. We note that the linearized TNMG has a linearized Weyl (conformal) invariance as the CSG does show [6].

In this paper, we explicitly show that a massive spin-2 mode is propagating on the flat spacetime when introducing the TNMG. Furthermore, we observe how the BMS/GCA correspondence is realized in the TNMG.

\section{TNMG IN FLAT SPACETIME}

We start with the TNMG action

$$
\begin{aligned}
& I_{\mathrm{TNMG}}=I_{\mathrm{CSG}}+I_{\mathrm{K}}, \\
& I_{\mathrm{CSG}}=\frac{1}{2 \kappa^{2} \mu} \int d^{3} x \sqrt{-g} \epsilon^{\lambda \mu \nu} \Gamma_{\lambda \sigma}^{\rho}\left(\partial_{\mu} \Gamma_{\rho \nu}^{\sigma}+\frac{2}{3} \Gamma_{\mu \tau}^{\sigma} \Gamma_{\nu \rho}^{\tau}\right), \\
& I_{\mathrm{K}}=\frac{1}{\kappa^{2} m^{2}} \int d^{3} x \sqrt{-g}\left(R^{\mu \nu} R_{\mu \nu}-\frac{3}{8} R^{2}\right),
\end{aligned}
$$

where $\kappa^{2}=16 \pi G, G$ is the Newton constant, $\mu$ the Chern-Simons coupling, and $m^{2}$ a mass parameter. We note that the GMG action is given by [7, 8$]$

$$
I_{\mathrm{GMG}}=\frac{1}{16 \pi G} \int d^{3} x \sqrt{-g}\left(\sigma R-2 \Lambda_{0}\right)+I_{\mathrm{TNMG}},
$$

where the TNMG is recovered in the limits of $\sigma \rightarrow 0$ and $\Lambda_{0}=-1 / \ell^{2} \rightarrow 0$. The equation of motion of the TNMG action is given by

$$
\frac{1}{\mu} C_{\mu \nu}+\frac{1}{2 m^{2}} K_{\mu \nu}=0,
$$


where the Cotton tensor $C_{\mu \nu}$ takes the form

$$
C_{\mu \nu}=\epsilon_{\mu}^{\alpha \beta} \nabla_{\alpha}\left(R_{\beta \nu}-\frac{1}{4} g_{\beta \nu} R\right),
$$

and the tensor $K_{\mu \nu}$ is given by

$$
\begin{aligned}
K_{\mu \nu} & =2 \nabla^{2} R_{\mu \nu}-\frac{1}{2} \nabla_{\mu} \nabla_{\nu} R-\frac{1}{2} \nabla^{2} R g_{\mu \nu}+4 R_{\mu \rho \nu \sigma} R^{\rho \sigma} \\
& -\frac{3}{2} R R_{\mu \nu}-g_{\mu \nu} R_{\rho \sigma} R^{\rho \sigma}+\frac{3}{8} R^{2} g_{\mu \nu} .
\end{aligned}
$$

As a solution to Eq. (77), let us choose the Minkowski spacetime expressed in terms of the outgoing EF coordinates

$$
d s_{\mathrm{EF}}^{2}=\bar{g}_{\mu \nu} d x^{\mu} d x^{\nu}=-d u^{2}-2 d r d u+r^{2} d \theta^{2}
$$

where $u=t-r$ is a retarded time. Considering the perturbation $h_{\mu \nu}$ around the EF background $\bar{g}_{\mu \nu}$

$$
g_{\mu \nu}=\bar{g}_{\mu \nu}+h_{\mu \nu}
$$

the linearized equation of Eq. (7) takes the form

$$
\frac{1}{\mu} \delta C_{\mu \nu}+\frac{1}{2 m^{2}} \delta K_{\mu \nu}=0
$$

Now, we consider the transverse and traceless conditions to select a massive mode propagating on the EF background as

$$
\bar{\nabla}^{\mu} h_{\mu \nu}=0, \quad h_{\mu}^{\mu}=0
$$

Then, we have the linearized fourth-order equation of motion as

$$
\epsilon_{\mu}^{\alpha \beta} \bar{\nabla}_{\alpha} \bar{\nabla}^{2}\left(\delta_{\beta}^{\rho}+\frac{\mu}{m^{2}} \epsilon_{\beta}^{\sigma \rho} \bar{\nabla}_{\sigma}\right) h_{\rho \nu}=0
$$

where the mass of the graviton is identified with $M=m^{2} / \mu$. Furthermore, this equation can be expressed compactly

$$
\left(D^{3} D^{M} h\right)_{\mu \nu}=0
$$

by introducing two mutually commuting operators as

$$
D_{\mu}^{\beta}=\epsilon_{\mu}^{\alpha \beta} \bar{\nabla}_{\alpha}, \quad\left(D^{M}\right)_{\mu}^{\beta}=\delta_{\mu}^{\beta}+\frac{\mu}{m^{2}} \epsilon_{\mu}^{\alpha \beta} \bar{\nabla}_{\alpha}
$$


Now, let us solve the first-order massive equation

$$
\left(D^{M} h^{M}\right)_{\mu \nu}=h_{\mu \nu}^{M}+\frac{\mu}{m^{2}} \epsilon_{\mu}^{\alpha \beta} \bar{\nabla}_{\alpha} h_{\beta \nu}^{M} \equiv(\mathrm{EOM})_{(\mu \nu)}=0
$$

directly. This will be done by assuming a proper ansatz

$$
h_{\mu \nu}^{M}(u, r, \theta)=f(\theta) G(u, r)\left(\begin{array}{ccc}
0 & 0 & 0 \\
0 & F_{r r}(r) & F_{r \theta}(r) \\
0 & F_{r \theta}(r) & F_{\theta \theta}(r)
\end{array}\right) .
$$

Then, the traceless condition of $h^{\mu}{ }_{\mu}=0$ takes the form

$$
r^{2} F_{r r}+F_{\theta \theta}=0
$$

while the transverse conditions $\bar{\nabla}^{\mu} h_{\mu \nu}=0$ lead to

$$
\begin{aligned}
& 0=F_{\theta \theta} G f^{\prime}+r f\left[r G F_{r \theta}^{\prime}+F_{r \theta}\left(G+r \partial_{r} G-r \partial_{u} G\right)\right] \\
& 0=F_{r \theta} G f^{\prime}+f\left[\frac{G}{r}\left(r^{3} F_{r r}^{\prime}-F_{\theta \theta}\right)+r F_{r r}\left(G+r \partial_{r} G-r \partial_{u} G\right)\right],
\end{aligned}
$$

for $\nu=\theta, r$, respectively, and for $\nu=u$, it vanishes. Here the prime $\left({ }^{\prime}\right)$ denotes the differentiation with respect to its argument.

The nine equations of motion take the following forms:

$$
\begin{aligned}
& 0=(\mathrm{EOM})_{(11)}=(\mathrm{EOM})_{(21)}=(\mathrm{EOM})_{(31)} \\
& 0=(\mathrm{EOM})_{(12)}=-r F_{r r} G f^{\prime}+f\left[r G F_{r \theta}^{\prime}+F_{r \theta}\left(G+r \partial_{r} G-r \partial_{u} G\right)\right] \\
& 0=(\mathrm{EOM})_{(13)}=r F_{r \theta} G f^{\prime}+f\left[r^{2} G F_{r r}-r G F_{\theta \theta}^{\prime}+F_{\theta \theta}\left(G-r \partial_{r} G+r \partial_{u} G\right)\right] \\
& \left.0=(\mathrm{EOM})_{(22)}=m^{2} r^{2} F_{r r} G f+\mu G\left[-r F_{r r} f^{\prime}+f\left(F_{r \theta}+r F_{r \theta}^{\prime}\right)+\mu r f F_{r \theta} \partial_{r} G\right)\right] \\
& 0=(\mathrm{EOM})_{(23)}=\mu r f F_{\theta \theta} \partial_{r} G-G\left[\mu r F_{r \theta} f^{\prime}+f\left(\mu r^{2} F_{r r}+\mu F_{\theta \theta}-r^{2}\left(m^{2} r F_{r \theta}+\mu F_{\theta \theta}^{\prime}\right)\right)\right] \\
& 0=(\mathrm{EOM})_{(32)}=F_{r \theta} G-\frac{\mu r}{m^{2}} F_{r r} \partial_{u} G \\
& 0=(\mathrm{EOM})_{(33)}=F_{\theta \theta} G-\frac{\mu r}{m^{2}} F_{r \theta} \partial_{u} G
\end{aligned}
$$

with $(u, r, \theta)=(1,2,3)$. From Eq. (25), one finds the relation

$$
F_{r \theta}=\frac{\mu r}{m^{2}} \frac{\partial_{u} G}{G} F_{r r}
$$

Also, from Eq. (26), one obtains the relation

$$
F_{\theta \theta}=\frac{\mu r}{m^{2}} \frac{\partial_{u} G}{G} F_{r \theta}=\frac{\mu^{2} r^{2}}{m^{4}} \frac{\left(\partial_{u} G\right)^{2}}{G^{2}} F_{r r} .
$$


Comparing this with the traceless condition (19), we have

$$
\left[\frac{\partial_{u} G}{G}\right]^{2}=-\left[\frac{m^{2}}{\mu}\right]^{2}
$$

which could be solved to give

$$
G(u, r)=C_{1}(r) e^{ \pm i \frac{m^{2}}{\mu} u}
$$

Choosing "-"sign, we obtain

$$
G(u, r)=C_{1}(r) e^{-i \frac{m^{2}}{\mu} u}, F_{r \theta}=-i r F_{r r}, F_{\theta \theta}=-r^{2} F_{r r}
$$

Using these relations, Eqs. (21)-(24) reduce to a single equation

$$
0=\mu r f C_{1}(r) F_{r r}^{\prime}-\left[i \mu C_{1}(r) f^{\prime}-\left\{\left(2 \mu+i m^{2} r\right) C_{1}(r)+\mu r C_{1}^{\prime}(r)\right\} f\right] F_{r r}
$$

which has a solution

$$
F_{r r}=\frac{e^{-i \frac{m^{2}}{\mu} r} r^{\frac{i f^{\prime}}{f}-2}}{C_{1}(r)} .
$$

Again, using this, Eqs. (21)-(24) become a single equation for $f(\theta)$

$$
\left[f^{\prime}(\theta)\right]^{2}=f(\theta) f^{\prime \prime}(\theta)
$$

whose solution is given by

$$
f(\theta)=e^{C_{2} \theta}
$$

with an undermined constant $C_{2}$.

As a result, we arrive at a solution

$$
h_{\mu \nu}^{M}(u, r, \theta)=e^{-i \frac{m^{2}}{\mu}(u+r)} e^{C_{2} \theta} r^{i C_{2}-2}\left(\begin{array}{ccc}
0 & 0 & 0 \\
0 & 1 & -i r \\
0 & -i r & -r^{2}
\end{array}\right)
$$

with $u+r=t$. We note that $C_{1}(r)$ disappears in Eq. (36), suggesting that one may choose $G(u)$ initially, instead of $G(u, r)$ in Eq. (18).

Importantly, when solving the first-order massive equation (17), one could not determine $C_{2}$. However, if one introduces the $2 \mathrm{D}$ GCA representations, it could be fixed to be $C_{2}=-i \xi$, implying that the integration constant $C_{2}$ can be interpreted as the eigenvalue of $L_{0}$ of the asymptotic BMS algebra in Minkowski spacetime. This may be taken as a hint that the 
TNMG provides a possible realization of the BMS algebra in three dimensions. Making the choice of $C_{2}=-i \xi$, we have the solution

$$
h_{\mu \nu}^{M}(u, r, \theta)=e^{-i \frac{m^{2}}{\mu}(u+r)} e^{-i \xi \theta} r^{\xi-2}\left(\begin{array}{ccc}
0 & 0 & 0 \\
0 & 1 & -i r \\
0 & -i r & -r^{2}
\end{array}\right),
$$

which is regarded as our main result.

In order to confirm that $h_{\mu \nu}^{M}$ satisfies the full equation (15), we apply the massless operator $D n$-times on $h_{\mu \nu}^{M}$ as

$$
\left(D^{n} h^{M}\right)_{\mu \nu}=\left(-\frac{m^{2}}{\mu}\right)^{n} h_{\mu \nu}^{M} .
$$

Using Eq. (38), it is easy to check that $h_{\mu \nu}^{M}$ satisfies the linearized fourth-order equation (15) as

$$
\left(D^{3} D^{M} h^{M}\right)_{\mu \nu}=\left(D^{3} h^{M}\right)_{\mu \nu}+\frac{\mu}{m^{2}}\left(D^{4} h^{M}\right)_{\mu \nu}=0 .
$$

\section{BMA/GCA CORRESPONDENCE}

We need more works to confirm that the TNMG provides a possible realization of the BMS algebra in three dimensions. In this direction, we may show that the BMS central charges are defined in the TNMG. We might interpret (37) to be one-parameter deformation of the CSG representation of the the BMS algebra, parametrized by $M=m^{2} / \mu$. This is because $h_{\mu \nu}^{M}(u, r, \theta) \sim e^{-i M(u+r)} h_{\mu \nu}^{\xi}(r, \theta)$ where $h_{\mu \nu}^{\xi}(r, \theta)$ is the CSG wave function in (2). Hence, we have to look for the BMS central charges $c_{1}$ and $c_{2}$, eigenvalue $\xi$ of operator $L_{0}$ and eigenvalue $\Delta$ of operator $M_{0}$.

In order to see what is going on the BMS/GCA correspondence in the TNMS, we first consider "the AdS/CFT correspondence on the $\mathrm{AdS}_{3}$ and its boundary" within the GMG (6). Two central charges of the GMG on the boundary are given by [12 14]

$$
\begin{aligned}
& c_{L}=\frac{3 \ell}{2 G}\left(\sigma+\frac{1}{2 m^{2} \ell^{2}}-\frac{1}{\mu \ell}\right), \\
& c_{R}=\frac{3 \ell}{2 G}\left(\sigma+\frac{1}{2 m^{2} \ell^{2}}+\frac{1}{\mu \ell}\right) .
\end{aligned}
$$

Taking two limits of $\sigma \rightarrow 0$ and $\ell \rightarrow \infty$ to obtain the TNMG, the corresponding BMS central charges are defined to be

$$
c_{1}=\lim _{\sigma \rightarrow 0, \ell \rightarrow \infty}\left(c_{R}-c_{L}\right)=\frac{3}{G \mu},
$$




$$
c_{2}=\lim _{\sigma \rightarrow 0, \ell \rightarrow \infty} \frac{c_{R}+c_{L}}{\ell}=0
$$

Here we observe the disappearance of $m^{2}$ in $c_{2}$ (43), which might not be a good news. In defining $c_{1,2}$, we have used the convention of ultra-relativistic limit in [4, 15, 16], which is opposite to $c_{1}$ and $c_{2}$ in the original convention of non-relativistic limit [17, 18]. The former convention is better to take the flat-spacetime limit from the $\mathrm{AdS}_{3}$ spacetime. Eqs. (42) and (43) show clearly that the BMS central charges are determined by the CSG (44) solely, implying that the central extensions are unaffected by the presence of $I_{\mathrm{K}}$-term (5). This explains why we have chosen $C_{2}=-i \xi$ in deriving the massive wave solution (37).

Now let us determine which one of the rigidity (weight) $\xi$ and scaling dimension $\triangle$ is related to the deformed parameter, mass $M=m^{2} / \mu$ of the graviton. Since these are eigenvalues as shown in

$$
L_{0}|\triangle, \xi>=\xi| \triangle, \xi>, \quad M_{0}|\triangle, \xi>=\triangle| \triangle, \xi>
$$

they are defined by

$$
\xi=\lim _{\ell \rightarrow \infty, \sigma \rightarrow 0}(h-\bar{h}), \triangle=\lim _{\ell \rightarrow \infty, \sigma \rightarrow 0} \frac{h+\bar{h}}{\ell} .
$$

Note that Eq. (44) can be understood as acting two operators $L_{0}=i \partial_{\theta}$ and $M_{0}=i \partial_{t}$ on the solution (37), defined in Ref. [3]. Here two weights $h$ and $\bar{h}$ are defined as the highest weight conditions of the GMG on the $\mathrm{AdS}_{3}: \mathcal{L}_{0}\left|\psi_{\mu \nu}>=h\right| \psi_{\mu \nu}>$ and $\overline{\mathcal{L}}_{0}\left|\psi_{\mu \nu}>=\bar{h}\right| \psi_{\mu \nu}>$. Then, $h$ and $\bar{h}$ are determined to be [19]

$$
(h, \bar{h})=\left(\frac{3+\ell m_{1}}{2}, \frac{-1+\ell m_{1}}{2}\right)
$$

where the mass is given by

$$
m_{1}=\frac{m^{2}}{2 \mu}+\sqrt{\frac{1}{2 \ell^{2}}-\sigma m^{2}+\frac{m^{4}}{4 \mu^{2}}} .
$$

According to the ultra-relativistic convention [15, 16], the connection between the GCA and the Virasoro algebras is given by

$$
L_{n}=\mathcal{L}_{n}-\overline{\mathcal{L}}_{-n}, \quad M_{n}=\frac{\mathcal{L}_{n}+\overline{\mathcal{L}}_{-n}}{\ell} .
$$

After a computation, one finds that

$$
\xi=2, \quad \triangle=\frac{m^{2}}{\mu} .
$$


The eigenvalue $\xi=2$ arises because it represents spin- 2 excitations. In the limit of $\mu \rightarrow \infty$, $\triangle \rightarrow 0$ as recovering the massless NMG. Using these, the massive wave solution (37) respects that of the GMG on the $\mathrm{AdS}_{3}$ as

$$
\tilde{h}_{\mu \nu}^{M}(u, r, \theta)=e^{-i \frac{m^{2}}{\mu}(u+r)-2 i \theta}\left(\begin{array}{ccc}
0 & 0 & 0 \\
0 & 1 & -i r \\
0 & -i r & -r^{2}
\end{array}\right) .
$$

At this stage, we note again that the central charges $c_{1}$ and $c_{2}$ in Eqs. (42) and (43) remain intact in compared to the CSG, but the scaling dimension $\triangle$ was changed from 0 to $m^{2} / \mu$. Thinking that the TNMG is one-parameter deformation of the CSG representation of the BMS algebra, one might expect that their central charges also be deformed. In order to explore this idea, we observe $c_{L / R}$ in (40) and (41) carefully. Considering the flat-spacetime limit from the $\mathrm{AdS}_{3}$ spacetime, one possibility is to consider the case

$$
\tilde{c}_{2}=\lim _{\sigma \rightarrow 0, \ell \rightarrow \infty} \ell\left(c_{L}+c_{R}\right)=\frac{3}{2 G m^{2}}
$$

while $c_{1}$ remains the same as in Eq. (42). This also requires a modification of the generator $\tilde{M}_{n}$ as

$$
\tilde{M}_{n}=\ell\left(\mathcal{L}_{n}+\overline{\mathcal{L}}_{-n}\right)
$$

instead of $M_{n}$ in (48). In this case, the flat-spacetime definition of the scaling dimension is changed to be

$$
\tilde{\triangle}=\lim _{\ell \rightarrow \infty, \sigma \rightarrow 0} \ell(h+\bar{h})
$$

while the rigidity $\xi$ remains unchanged. For the TNMG, this lead to the infinity as

$$
\tilde{\triangle}=\lim _{\ell \rightarrow \infty, \sigma \rightarrow 0} \ell\left(1+\ell m_{1}\right) \rightarrow \infty
$$

which cannot be acceptable. As a result, the BMS charge $\tilde{c}_{2}$ seems to be unphysical, even though it has a finite value (51) in the flat-spacetime limit.

\section{GMG SOLUTION IN THE FLAT-SPACETIME LIMT}

Now, it is very important to know what waveform of the GMG [12] provides (50) in the flat-spacetime limit. Directly, this task will determine which one between $\left(c_{2}=0, \Delta=\right.$ 
$\left.m^{2} / \mu\right)$ and $\left(\tilde{c}_{2}=\frac{3}{2 G m^{2}}, \tilde{\Delta}=\infty\right)$ is correct. In particular, the GMG wave solution for the left-moving massive graviton is described by

$$
\psi_{\mu \nu}^{\mathrm{L}}\left(\rho, \tau^{+}, \tau^{-}\right)=f\left(\rho, \tau^{+}, \tau^{-}\right)\left(\begin{array}{ccc}
1 & 0 & \frac{2 i}{\sinh (2 \rho)} \\
0 & 0 & 0 \\
\frac{2 i}{\sinh (2 \rho)} & 0 & -\frac{4}{\sinh ^{2}(2 \rho)}
\end{array}\right)
$$

in the light-cone coordinates of $\left(\rho, \tau^{ \pm}=\tau \pm \phi\right)$ for the $\mathrm{AdS}_{3}$ spacetime. Here the amplitude $f$ is given by

$$
f\left(\rho, \tau^{+}, \tau^{-}\right)=e^{-i h \tau^{+}-i \bar{h} \tau^{-}}(\cosh \rho)^{-(h+\bar{h})} \sinh ^{2} \rho,
$$

where two weights $h$ and $\bar{h}$ are already given by (46). We note that $\psi_{\mu \nu}^{\mathrm{L}}$ satisfies the traceless and transverse conditions: $\psi_{\mu}^{\mathrm{L} \mu}=0, \bar{\nabla}_{\mu} \psi^{\mathrm{L} \mu \nu}=0$. As is suggested in Ref. [4], we express the EF coordinates in terms of global coordinates

$$
u=\ell(\tau-\rho), \quad r=\ell \rho, \quad \theta=\phi .
$$

Then, we have a transformed tensor mode

$$
\psi_{\mu \nu}^{\mathrm{L}}(u, r, \theta)=f(u, r, \theta)\left(\begin{array}{ccc}
1 & 1+\frac{2 i}{\sinh \left(\frac{2 r}{\ell}\right)} & \ell \\
1+\frac{2 i}{\sinh \left(\frac{2 r}{\ell}\right)} & 1+\frac{4 i}{\sinh \left(\frac{2 r}{\ell}\right)}-\frac{4}{\sinh ^{2}\left(\frac{2 r}{\ell}\right)} & \left(1+\frac{2 i}{\sinh \left(\frac{2 r}{\ell}\right)}\right) \ell \\
\ell & \left(1+\frac{2 i}{\sinh \left(\frac{2 r}{\ell}\right)}\right) \ell & \ell^{2}
\end{array}\right),
$$

where the transformed amplitude takes the form

$$
f(u, r, \theta)=e^{-i\left(\frac{h+\bar{h}}{\ell}\right)(u+r)-i(h-\bar{h}) \theta}\left[\cosh \left(\frac{r}{\ell}\right)\right]^{-(h+\bar{h})} \sinh ^{2}\left(\frac{r}{\ell}\right) .
$$

Thus, taking the flat-spacetime limit of $\ell \rightarrow \infty$ while keeping $u$ and $r$ finite, and making use of $\Delta$ in (45) (but not $\tilde{\Delta}$ in (153) $)$, we arrive at

$$
\psi_{\mu \nu}^{\mathrm{L}}(u, r, \theta) \simeq e^{-i \frac{m^{2}}{\mu}(u+r)-2 i \theta}\left(\begin{array}{ccc}
0 & 0 & 0 \\
0 & 1 & -i r \\
0 & -i r & -r^{2}
\end{array}\right),
$$

which is exactly the same form of (50). This proves that the massive wave solution (50) represents a truly massive graviton mode propagating in the Minkowski spacetime background. In this case, $c_{2}=0\left(\Delta=m^{2} / \mu\right)$ is a correct BMS representation for the TNMG. Finally, we wish to stress that the TNMS provides one-parameter $\left(m^{2} / \mu\right)$ deformation of the CSG representation of the the BMS algebra. However, the central charges $\left(c_{1}, c_{2}\right)$ are not affected by this deformation but the scaling dimension $\Delta$ is changed. 


\section{DISCUSSIONS}

Our work was inspired by the observation that even though the CSG has no local degrees of freedom, it provides the first evidence for a holographic correspondence (the BMS/CFT correspondence) [4]. Its dual field theory is considered as a chiral CFT with a central charge of $c=24$.

In order to see what happens for the holographic properties of a gravitational theory with a local degree of freedom, we have investigated the TNMG in the Minkowski spacetime. Solving the first-order massive equation (17) together with the traceless and transverse conditions, we have found a massive wave solution (37). Concerning the BMS/GCA correspondence in the TNMG, we have $c_{1}=\frac{3}{G \mu}$ and $c_{2}=0$ as in the CSG. This means that the NMG-term $\left(I_{\mathrm{K}}\right)$ does not contribute to the central charge of the boundary field theory. Also we have the same rigidity $\xi=2$ as in the CSG [20] where $(h, \bar{h})=\left(\frac{3+\ell \mu}{2}, \frac{-1+\ell \mu}{2}\right)$, but a different scaling dimension $\Delta=m^{2} / \mu$ from $\Delta=\mu$ of the CSG. Here, some difference arises in defining $\Delta$ : in Ref. [3], $\Delta=0$ for the CSG because they have taken the scaling limit of $\mu \rightarrow 0$. However, in this work, we did not require the scaling limit of $\mu \rightarrow 0, G \rightarrow \infty$, but use the flat spacetime limit of $\sigma \rightarrow 0, \ell \rightarrow \infty$ to get the TNMG. Importantly, we have obtained the massive graviton wave solution (50) which is recovered from the GMG-wave solution when taking the flat spacetime limit and using $\xi=2$ and $\Delta=m^{2} / \mu$.

We discuss asymptotically flat boundary condition on the wave solution (50). Actually, there is a difference between the CSG and the TNMG because there is a change in the radial solution between $h_{\mu \nu}^{\xi}(\underline{21})$ and $\tilde{h}_{\mu \nu}^{M}(\underline{50}): \tilde{h}_{\mu \nu}^{M}$ is regular in the interior, but incompatible with the asymptotically flat boundary condition (3) in Ref. [4]. Therefore, there is a little improvement on the radial boundary condition of a massive graviton mode.

Consequently, we have shown that the TNMG has a single massive mode propagating on the flat spacetime, whereas there is no physically propagating degrees of freedom from the CSG. This means that the TNMS provides one-parameter deformation of the CSG representation of the the BMS algebra, parametrized by $m^{2} / \mu$. However, their central charges $\left(c_{1}, c_{2}\right)$ are unaffected by this deformation, but the scaling dimension $\Delta$ is changed. 


\section{Acknowledgments}

We would like to thank D. Grumiller for helpful discussions. This work was supported by the National Research Foundation of Korea (NRF) grant funded by the Korea government (MEST) through the Center for Quantum Spacetime (CQUeST) of Sogang University with grant number 2005-0049409. Y. S. Myung was also supported by the National Research Foundation of Korea (NRF) grant funded by the Korea government (MEST) (No.2012040499). Y.-J. Park was also supported by World Class University program funded by the Ministry of Education, Science and Technology through the National Research Foundation of Korea(No. R31-20002).

[1] J. M. Maldacena, Adv. Theor. Math. Phys. 2, 231 (1998) hep-th/9711200.

[2] J. D. Brown and M. Henneaux, Commun. Math. Phys. 104, 207 (1986).

[3] A. Bagchi, Phys. Rev. Lett. 105, 171601 (2010) arXiv:1006.3354 [hep-th]].

[4] A. Bagchi, S. Detournay and D. Grumiller, Phys. Rev. Lett. 109, 151301 (2012) arXiv:1208.1658 [hep-th]].

[5] G. Barnich and G. Compere, Class. Quant. Grav. 24, F15 (2007) gr-qc/0610130].

[6] R. Andringa, E. A. Bergshoeff, M. de Roo, O. Hohm, E. Sezgin and P. K. Townsend, Class. Quant. Grav. 27, 025010 (2010) arXiv:0907.4658 [hep-th]].

[7] E. A. Bergshoeff, O. Hohm and P. K. Townsend, Phys. Rev. Lett. 102, 201301 (2009) arXiv:0901.1766 [hep-th]].

[8] E. A. Bergshoeff, O. Hohm and P. K. Townsend, Phys. Rev. D 79, 124042 (2009) arXiv:0905.1259 [hep-th]].

[9] Y. S. Myung, arXiv:1212.6805 [gr-qc].

[10] D. Dalmazi and E. L. Mendonca, JHEP 0909, 011 (2009) [arXiv:0907.5009 [hep-th]].

[11] O. Hohm, A. Routh, P. K. Townsend and B. Zhang, Phys. Rev. D 86, 084035 (2012) arXiv:1208.0038 [hep-th]].

[12] Y. Liu and Y. -W. Sun, Phys. Rev. D 79, 126001 (2009) [arXiv:0904.0403 [hep-th]].

[13] E. A. Bergshoeff, S. de Haan, W. Merbis, J. Rosseel and T. Zojer, Phys. Rev. D 86, 064037 (2012) arXiv:1206.3089 [hep-th]]. 
[14] Y. -W. Kim, Y. S. Myung and Y. -J. Park, Phys. Rev. D 86, 064017 (2012) arXiv:1207.3149 [hep-th]].

[15] A. Bagchi, S. Detournay, R. Fareghbal and J. Simon, arXiv:1208.4372 [hep-th]].

[16] D. Grumiller, W. Riedler, J. Rosseel and T. Zojer, arXiv:1302.0280 [hep-th]].

[17] A. Bagchi, R. Gopakumar, I. Mandal and A. Miwa, JHEP 1008, 004 (2010) arXiv:0912.1090 [hep-th]].

[18] M. R. Setare and V. Kamali, Europhys. Lett. 98, 31001 (2012) [arXiv:1103.0457[ [hep-th]].

[19] D. Grumiller, N. Johansson and T. Zojer, JHEP 1101, 090 (2011) arXiv:1010.4449 [hep-th]].

[20] W. Li, W. Song and A. Strominger, JHEP 0804, 082 (2008) [arXiv:0801.4566 [hep-th]]. 